PAPER

\title{
Computational acoustic vision by solving phase ambiguity confusion
}

\author{
Ryuichi Shimoyama* and Ken Yamazaki \\ Department of Electrical and Electronic Engineering, \\ College of Industrial Technology, Nihon University, \\ Izumi-cho 1-2-1, Narashino, 275-8575 Japan
}

(Received 13 December 2007, Accepted for publication 31 October 2008)

\begin{abstract}
Computational acoustic vision by solving phase ambiguity confusion (CAVSPAC) is proposed for two-dimensional colorful imaging such as pointillisme in the broadband sound environment. The 2D distributions of equivalent point sources were identified as an image from the cross-power spectral phases of sound pressure measured by two pairs of microphones. Each point source was assigned a color corresponding to its frequency. Multiple source locations are introduced from one cross-spectral phase value because of "phase ambiguity" at high frequencies, when the microphone interval is wider than the sound wavelengths. The true source location was extracted from multiple source locations as being the frequency independent. The broadband noise source was visualized with a single two-way loudspeaker set at various positions in the reverberative room. Using CAVSPAC, the 2D image could be identified for the broadband sound source from all directions spherically, except in the area just beside, above and under the microphones. The moderate wider microphone interval than the sound wavelengths led to a better resolution at the source image.
\end{abstract}

Keywords: 2D, Acoustic source image, Phase ambiguity, Reverberant condition, Broadband

PACS number: 43.60.Pt, 43.66.Qp [doi:10.1250/ast.30.199]

\section{INTRODUCTION}

In view of the human recognition of the external sound environment, the concept of acoustic scene analysis has been introduced in recent years [1], wherein acoustic sound is treated analogously to an optical scene. An acoustic environment always surrounds us. By simply listening, we can recognize whether a speaker is standing on seashore or in the mountains. We can also distinguish the sound of waves on a seashore from that played by a tape recorder. Sounds other than the speaker's voice might help us to recognize the external sound environment in view of their sound quality and distribution in space. Several algorithms imitating animal or human auditory systems have been proposed for acoustic source localization using two microphones [2-7]. Since the computational algorithm for source localization using two microphones will provide important keys for clarifying the auditory mechanism with two ears. These algorithms were intended to be used to determine the main source directions for engineering applications, such as the localization of speakers. The surrounding sound was treated as noise to be eliminated in source localization. The external acoustic environment should be treated as a source

*e-mail: shimoyama.ryuichi@nihon-u.ac.jp distribution in space.

Imaging can provide us with more critical information on the acoustic environment. A two-dimensional microphone-array system can be used to visualize the acoustic sound pressure distribution, not the sound source distribution, at a certain frequency [8]. This distribution can be visualized only when the frequency is given in advance. The external sound environment is generally over a broadband of frequencies. Broadband source imaging using the microphone-array system requires several acoustic images to be obtained over the entire measurement range of frequencies [9]. For cases in which the acoustic environment was expressed as a number of distributed point sources, Wang et al. proposed point-source imaging, which represents each broadband source as a point source [10]. This algorithm requires an iterative process for identifying both the incident signals and their positions. Kamiakito et al. have shown that the point source distribution can express the broadband sound environment as an image based on the ray-trace model, in which each point source represents one sound source direction at a single frequency [11]. Each point source was localized using the crosspower spectral phase at that frequency, and the microphone interval was set shorter than the sound wavelength to avoid "phase ambiguity." "Phase ambiguity" leads to confusion 
in computational source localization, since multiple source locations are introduced from a cross-spectral phase value measured by two microphones at high frequencies, when the microphone interval is wider than the sound wavelengths. Konishi solved similar "phase ambiguity" confusion in time domain in the midbrain mechanism of the barn owl for auditory localization [12]. Shimoyama proposed a new ray-trace model, in which the phase ambiguity confusion was solved in the frequency domain, to localize the broadband sound sources at higher frequencies, even when the microphone interval was wider than the wavelengths [13]. The frequency domain model is suitable for simulating the midbrain mechanism, since the time difference may be transformed to a phase difference owing to the periodic nerve spikes transmitted from the cochlea to the midbrain.

In the present paper, computational acoustic vision by solving phase ambiguity confusion (CAVSPAC) is proposed for $2 \mathrm{D}$ colorful imaging in the broadband sound environment. The 2D distributions of equivalent point sources are identified as images from the ambiguous crosspower spectral phase values of sound pressure measured with two pairs of microphones. Each point source represents an equivalent sound source synthesizing all sound propagating from various directions at a single frequency. Such an image shows the external acoustic environment diagrammatically. The algorithm for CAVSPAC is fundamentally based on a frequency domain model modified from Konishi's model, rather than on a direct analogy to the barn owl auditory system. This is the $2 \mathrm{D}$ version of the previous model [13] in which the "phase ambiguity" confusion was solved without any iterative process. Each equivalent point source is newly assigned a color corresponding to its frequency. It can represent the frequency components of sound as a colorful tone composed of clustered dots, since we perceive the cluster of colorful dots not as independent ones but as a colorful tone and shape.

In Chap. 2, the principle of CAVSPAC is described. The localization procedure for each point source, such as measurement and multiple angle identification, is shown at a single frequency. Then, the imaging procedure on the broadband sound environment is described, including the determination of the $2 \mathrm{D}$ distribution of equivalent point sources, and the assignment of colors.

In Chap. 3, the 2D images of equivalent point sources are identified in the case that a single two-way loudspeaker is set at various positions in a reverberant room. True point sources are extracted from multiple sources introduced as a result of "phase ambiguity." The benefit of CAVSPAC is confirmed by comparing the 2D source images for the same loudspeaker for two different microphone intervals.

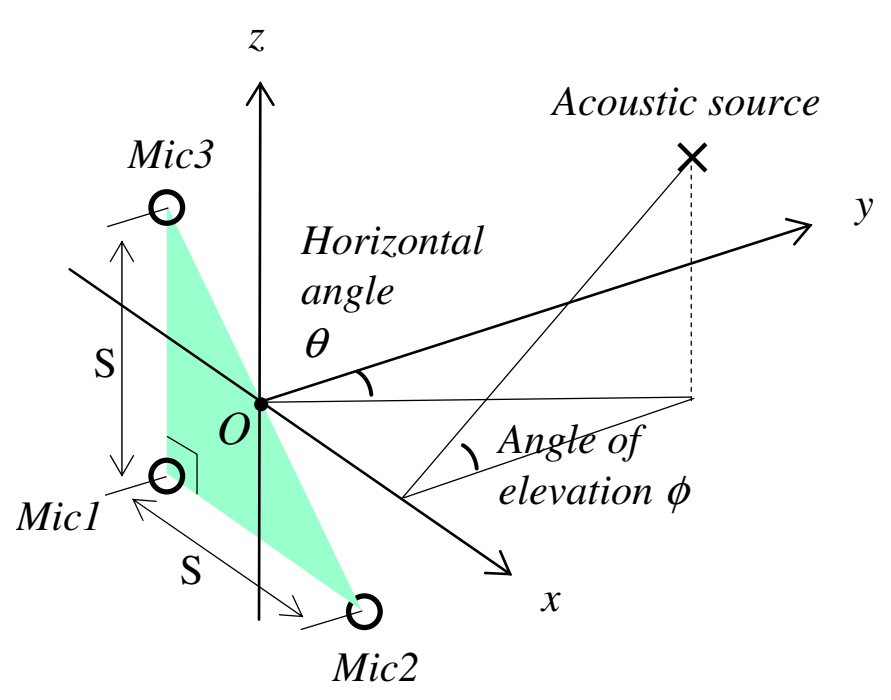

Fig. 1 Configuration of three sensors. Each sensor is aligned at an apex of a right triangle on the $x-z$ plane.

\section{METHOD AND PROCEDURES}

The 2D localization procedure for an equivalent point source at a single frequency is described. The 2D localization of a source requires at least two different directions, i.e., the horizontal and vertical directions. Figure 1 shows the configuration of sensors, Mic1, Mic2, and Mic3, and an acoustic source. The source positions are identified on two independent angles using two pairs of sensors, Mic1-Mic2 and Mic1-Mic3, where Mic1 is common to both pairs. First, a one-dimensional model is discussed for source localization using a pair of sensors, Mic1 and Mic2. Next, the extension of this one-dimensional model to two dimensions is introduced. Numerous point sources are plotted on the horizontal angle - angle of elevation plane and are assigned colors corresponding to frequencies for colorful source imaging over the full range of frequencies.

\subsection{Ambiguous Phase Differences and Source Angles}

It is well known that an object diffracts and reflects sound. The effects of both diffraction and reflection depend on the shape, size, material and surface condition of the objects. A simple ray-trace model of sound propagation is adopted for localizing the source without any a priori information on the objects in real environment. Figure 2 shows a pair of sensors positioned at a distance $D$ from a point source that radiates sound. If the path difference $\Delta d$ between $\mathrm{AE}$ and $\mathrm{BE}$ is known and the sensor interval $S$ is given in Fig. 2, the angle $\theta$ for the source can be calculated using the principles of plane geometry in Eqs. (1) and (2), when the angle $\theta$ for the source is numerically given.

$\Delta x=D \tan \theta \quad(\mathrm{m})$

$\Delta d=\sqrt{D^{2}+(S / 2+\Delta x)^{2}}-\sqrt{D^{2}+(S / 2-\Delta x)^{2}}$ 


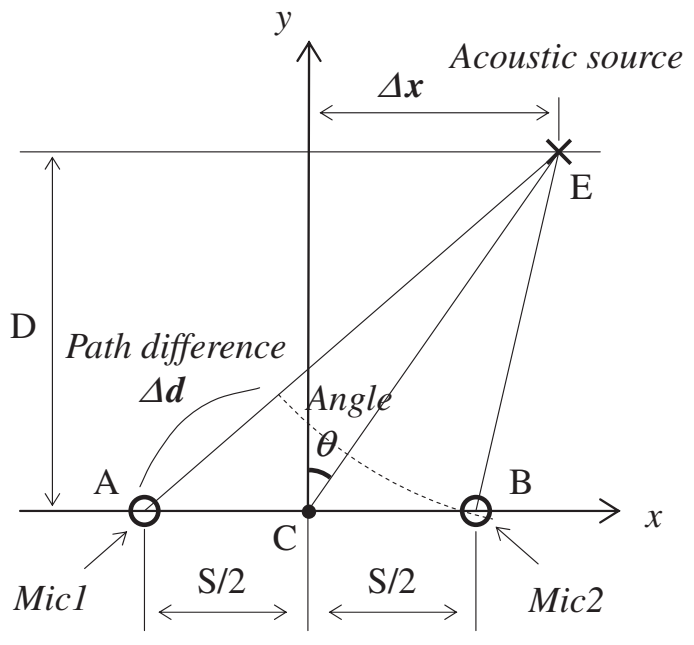

Fig. 2 Geometry of a pair of sensors and a source (plane figure).

It is not always necessary to measure the distance $D$ in advance because the distance $D$ from the sensors to the microphones has less than a $2 \%$ effect on the angle $\theta$ for the source when $D / S>3$ [7]. A one-to-one correspondence between the path difference and the angle for the source can be found only under conditions such that $D / S>3$. An arbitrary value, which is three times greater than $S$, should be substituted to approximate $D$ in Eqs. (1) and (2) when $D / S>3$. Since $\Delta d$ cannot be measured directly, we instead measure the phase difference $\Delta \phi$ of sound pressure, then indirectly estimate $\Delta d$ from it.

The phase difference of sound pressure measured by each sensor is expressed as

$$
\Delta \phi=\frac{\Delta d}{c} f \times 360 \quad(\mathrm{deg}),
$$

in which $c$ is the speed of sound, and $f$ is the frequency. "Phase ambiguity" prevents us from estimating an unique $\Delta d$ directly using Eq. (3). Figure 3 schematically shows "phase ambiguity," in which the sounds propagating from two different directions $\theta_{1}$ and $\theta_{2}$ have the same phase difference value at a frequency between Mic1 and Mic2, since $\Delta \phi$ can be replaced with $\Delta \phi+360$ in phase. Generally, the phase difference $\Delta \phi$ is replaced with $\Delta \phi+360 n$ :

$$
\Delta \phi \rightarrow \Delta \phi+360 n \quad(\mathrm{deg})
$$

in which $n$ is an integer. Substituting Eq. (4) for the phase difference $\Delta \phi$ in Eq. (3), we have

$$
\Delta d^{\prime}=\frac{c(\Delta \phi+360 n)}{360 f} \quad(\mathrm{~m}) .
$$

The path difference is defined ambiguously. That is, multiple path differences $\Delta d^{\prime}$ are given by the phase

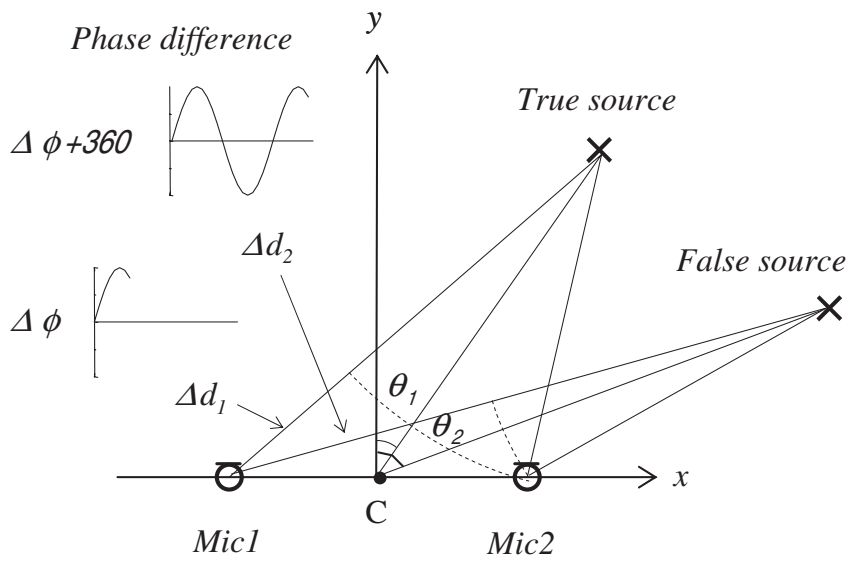

Fig. 3 Multiple source localization due to "phase ambiguity" at a single frequency.

difference value corresponding to integer $n$. The angles $\theta$ for the source can be estimated when the absolute value $\varepsilon$ of the residual in Eq. (6) is smaller than a threshold value, $\varepsilon_{0}$.

$$
\varepsilon=\left|\Delta d-\Delta d^{\prime}\right| \quad(\mathrm{m})
$$

As a result, the horizontal source angles $\theta$ are also identified as multiple values.

\subsection{D Colorful Source Imaging Procedures}

The 2D colorful source imaging procedures, which include five operations, are outlined in Fig. 4.

2.2.1. Measuring quasi-steady cross-power spectral phase

The quasi-steady cross-power spectral phase is measured as the phase difference over the range of frequencies. Each sound signal that is detected is multiplied by a Hanning window and is transformed to the frequency domain via a DFT algorithm. The output pairs in the frequency domain are multiplied to form the cross-power spectrum. The phase difference spectrum is obtained as the cross-power spectral phases overlapped and averaged over frequencies. Time averaging treats sound as quasistationary so as to emphasize the steady signals as a result of integration.

\subsubsection{Multiple angles localization}

Multiple angles are identified at each frequency. The value of the phase difference in Subsection 2.2.1 is substituted for $\Delta \phi$ in Eq. (5). Multiple angles for a target source are numerically calculated for several integer values $n=0, \pm 1, \pm 2, \ldots$ using Eqs. (1), (2), (5) and (6) over the range of \pm 90 degrees at an arbitrary frequency. The calculated angles are plotted as dots on the frequencysource angle plane. Frequency spectra of such angles indicate not only the acoustic source distribution in space, but also the frequency components of these angles for both true and false source locations. 


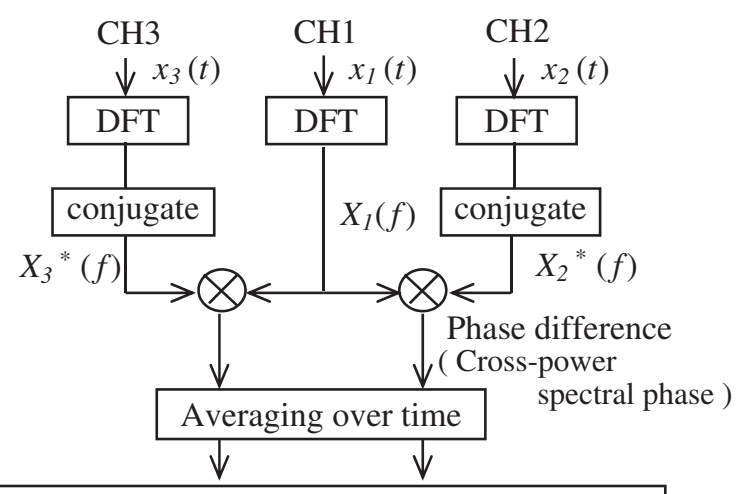

Multiple angles identification for target source using phase differences

Extraction of frequency-independent angles from multiple angles

$\downarrow \quad \downarrow$

Finding 2D location of point sources as combinations of two angles nearest to each frequency-independent angle

Assigning a color to each point source

Fig. 4 Proposed algorithm on CAVSPAC.

\subsubsection{D location as combinations of horizontal angles} and angles of elevation

The procedures described above are extended to two dimensions using the two pairs of microphones shown in Fig. 1. Multiple source angles can be identified independently at an arbitrary frequency, for both the horizontal angle and the angle of elevation. The $2 \mathrm{D}$ locations of point sources are numerically calculated as all combinations of two angles that are selected from multiple angles identified for each angle at an arbitrary frequency, over the range of \pm 90 degrees. The locations are expressed as $\left(\theta_{i}, \phi_{j}\right)$ at an arbitrary frequency when the horizontal angles $\theta_{i}$ $(i=1, \ldots, l)$ and the angles of elevation $\phi_{j}(j=1, \ldots, m)$ are ambiguously calculated. These locations are plotted as dots on the horizontal angle - angle of elevation plane over the frequency range. The point source distribution shows both the true and false source locations. The converged group of point sources is treated as the target, since the true location of a fixed source that radiates broadband sound is independent of frequency.

2.2.4. 2D location of true point source extracted from the multiple locations

The 2D location of the true point source is selected as a combination of two angles nearest to the frequencyindependent angles [13-15]. At each frequency, two angles to one equivalent center are estimated from the sound wavefronts, by the procedure described in the reference [13], under the hypothesis that sound propagates straight without diffraction or reflection. The obtained point source image may reflect this hypothesis.

\subsubsection{Assigning a color to each point source}

Each point source, which ideally radiates a single frequency tone, is assigned a color corresponding to its frequency. The cluster of colorful dots represents the distribution of frequency components of sound in space as a colorful tone, since dots in a cluster are perceived not as independent ones but as a colorful tone. The shape made by the colorful dots in clusters can also be perceived similarly to pointillisme, in which an optical scene is drawn with colorful dots.

\section{APPLICATIONS}

The 2D images of equivalent colorful sound sources for a single two-way loudspeaker were identified under several conditions. The room was approximately $11.2 \mathrm{~m}$ in length, $7.2 \mathrm{~m}$ in width, and $2.7 \mathrm{~m}$ in height, and the reverberation time was $0.5 \mathrm{~s}$. Three microphones (Type 4190, B\&K) were aligned in the same direction at $20 \mathrm{~cm}$ intervals (in Fig. 2, $S=0.2 \mathrm{~m}$ ) using the fitting on a tripod. The measurement was conducted using a multi-analyzer system (Pulse 3560C, B\&K). The two-way loudspeaker, which was composed of a tweeter and a woofer, was set at a height of $1 \mathrm{~m}$ at the center of the reverberant room. The tweeter was mounted $9 \mathrm{~cm}$ higher than the woofer on the baffle. The same white noise signal was played through each loudspeaker using a tape recorder. The sampling rate was $32.8 \mathrm{kHz}$. The frame period was set to $250 \mathrm{~ms}$ in the DFT procedure. The cross-power spectral phase was overlapped $75 \%$ and consisted of the average of ten measurements.

\subsection{D Source Image for Single Two-Way Loud- speaker}

The 2D image of equivalent colorful point sources for a two-way loudspeaker was identified. A single twoway loudspeaker was positioned exactly in front of the microphones at a distance of $1 \mathrm{~m}$ (in Fig. 2, $D=1 \mathrm{~m}$ ). Figures 5(a) and 5(b) show the phase difference spectra measured with two pairs of microphones: Mic1-Mic2 and Mic1-Mic3. Most of the phase difference values were distributed at approximately zero degrees in the case of the horizontal angle in Fig. 5(a). In Fig. 5(b), by contrast, the line slope representing the distribution of the cluster data became slightly negative at frequencies lower than $7 \mathrm{kHz}$ in the case of the angle of elevation. This negative slope corresponds to the slightly lower position of the woofer. Reverberation might scatter these values randomly, since the phase difference values were not distributed so widely in the case of similar measurements in the anechoic chamber [7]. Multiple angles for the loudspeaker were identified at frequencies higher than approximately $1.6 \mathrm{kHz}$ 


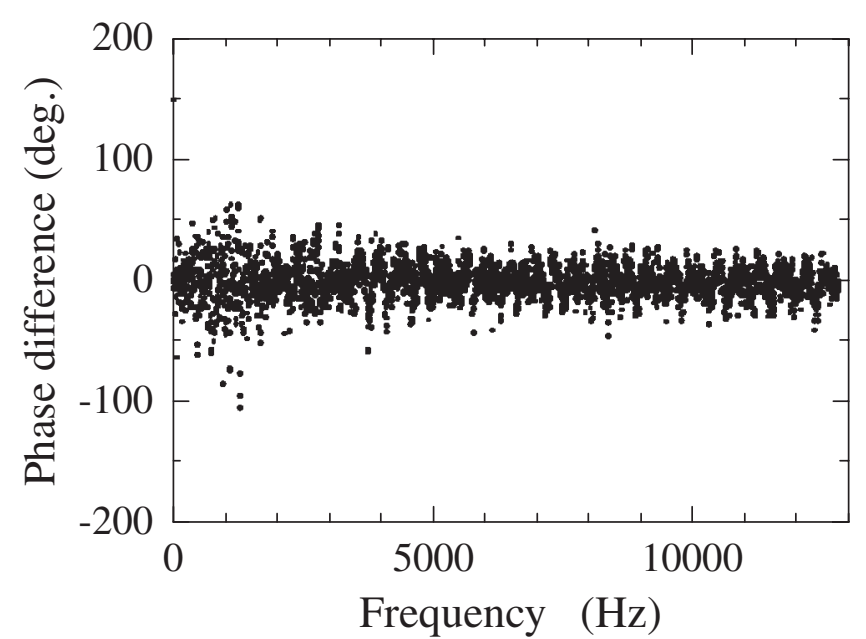

(a) Horizontal (Mic1 - Mic2)

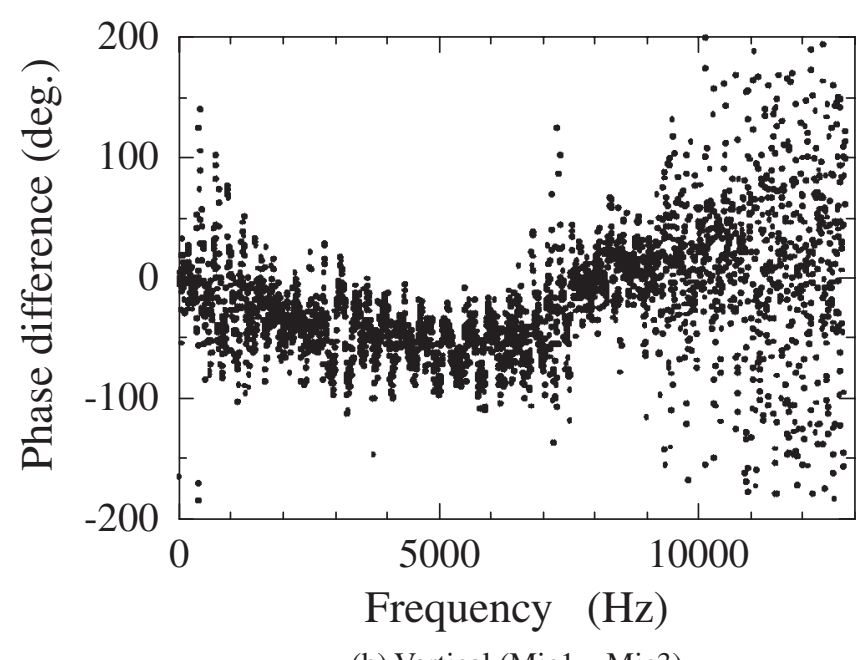

(b) Vertical (Mic1 - Mic3)

Fig. 5 Measured phase difference spectra of sound pressure (a) horizontally and (b) vertically (broadband noise from a single two-way loudspeaker in the reverberative room; $D=1 \mathrm{~m}, S=0.20 \mathrm{~m}$ ).

in Fig. 6, corresponding to Fig. 5. The angles were calculated for different integer values of $n$ in Eq. (5). Numerous curves representing the distribution of cluster data can be observed in both figures. Each curve may be described as the frequency spectrum of a source-identified angle. These curves are categorized as true or false by judging whether the angle is stable over the frequency range. In this case, only true angles should be distributed at approximately zero degree over the frequency range. The 2D image of equivalent colorful point sources for a single two-way loudspeaker is shown in Fig. 7. Each point source, which ideally radiates a single frequency tone, is assigned a color over the frequency range from $0.2 \mathrm{kHz}$ (red) to $12.8 \mathrm{kHz}$ (blue). Numerous point sources converged near the true loudspeaker location in which both the horizontal angle and angle of elevation were approximately zero degree. Several thick and thin lines, which indicate false

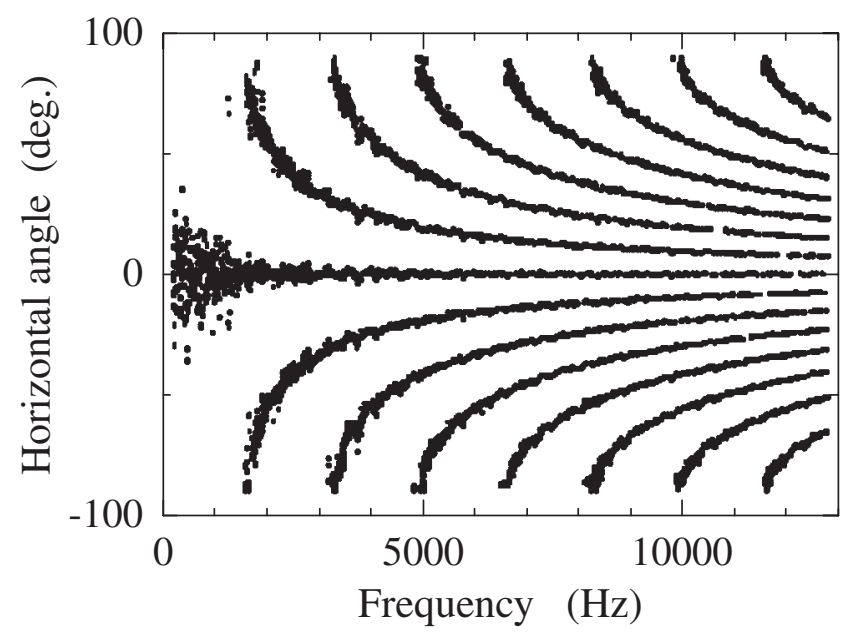

(a) Horizontal (Mic1 - Mic2)

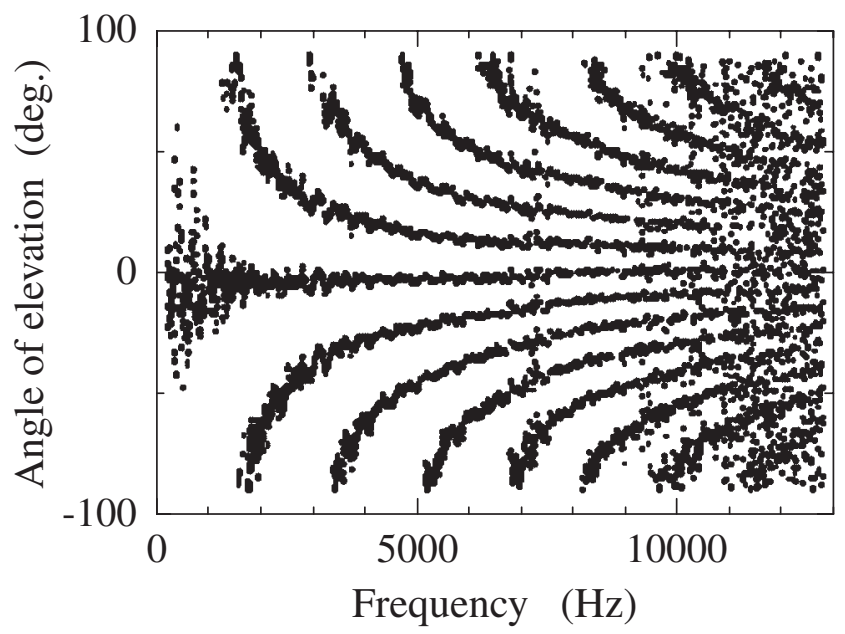

(b) Vertical (Mic1 - Mic3)

Fig. 6 Frequency spectra of multiple angles identified (a) horizontally and (b) vertically, corresponding to Fig. $5(D=1 \mathrm{~m}, S=0.20 \mathrm{~m})$.

point sources, are distributed radially from the loudspeaker position. This means that the false source location depends on the frequency. The source distribution generally appears to be bisymmetrical horizontally and unsymmetrical vertically with respect to the loudspeaker position, since two different types of loudspeakers radiate sound. The location at which the colorful point sources converged over the frequency range indicates the broadband source position.

\subsection{D Source Images for a Distant Loudspeaker}

The 2D images of equivalent colorful point sources were identified for a single two-way loudspeaker positioned at a distance of $3 \mathrm{~m}$ from the microphones. The loudspeaker was also set exactly in front of the microphones at the center of the reverberant room. The 2D colorful source image is shown in Fig. 8. The source image 


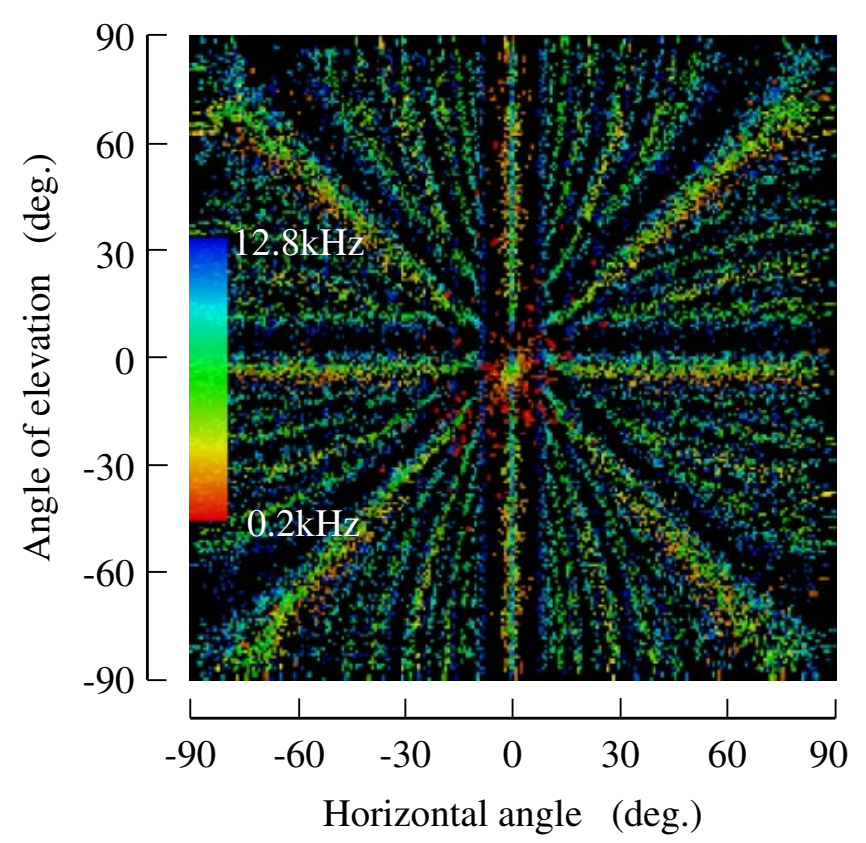

Fig. 7 2D image of colorful point sources equivalent to a single two-way loudspeaker exactly in front of the microphones. Colors are assigned to the frequency tones from $0.2 \mathrm{kHz}$ to $12.8 \mathrm{kHz}(D=1 \mathrm{~m}, S=0.20 \mathrm{~m})$.

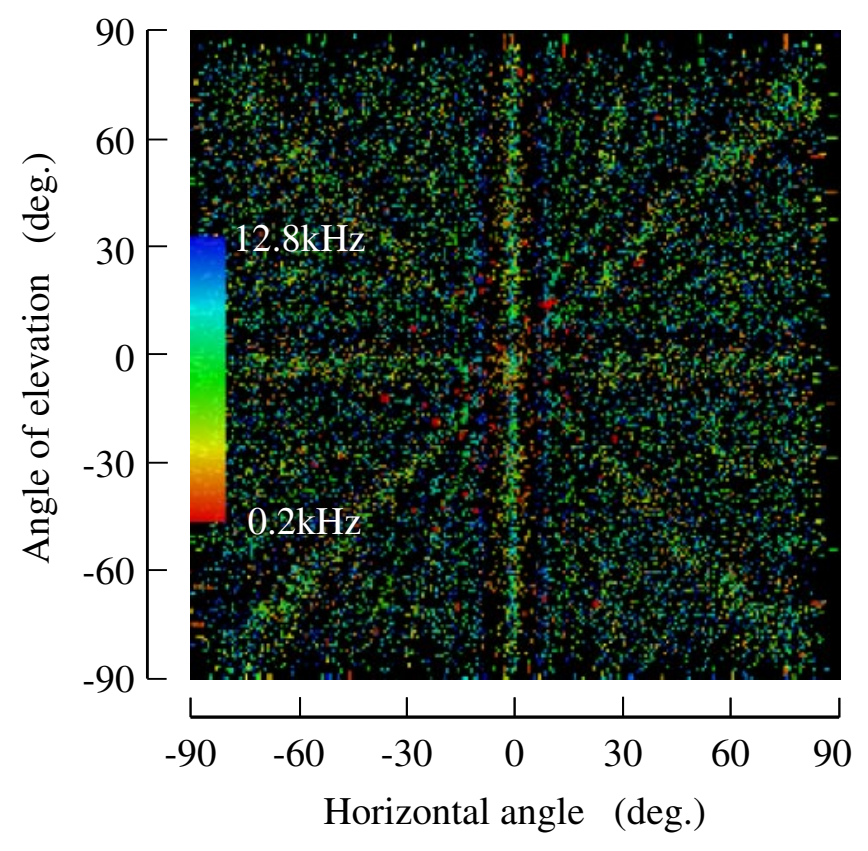

Fig. 8 2D image of colorful point sources equivalent to a single two-way loudspeaker $(D=3 \mathrm{~m}, S=0.20 \mathrm{~m})$.

was similar to that with $D=1 \mathrm{~m}$ (Fig. 7). The loudspeaker position could be observed at the crossing point of the false point source patterns on it. Several fine lines, composed of false point sources, disappeared in this case. This result indicates that the source image appears to become more obscure with increasing distance between the loudspeaker and the microphones.

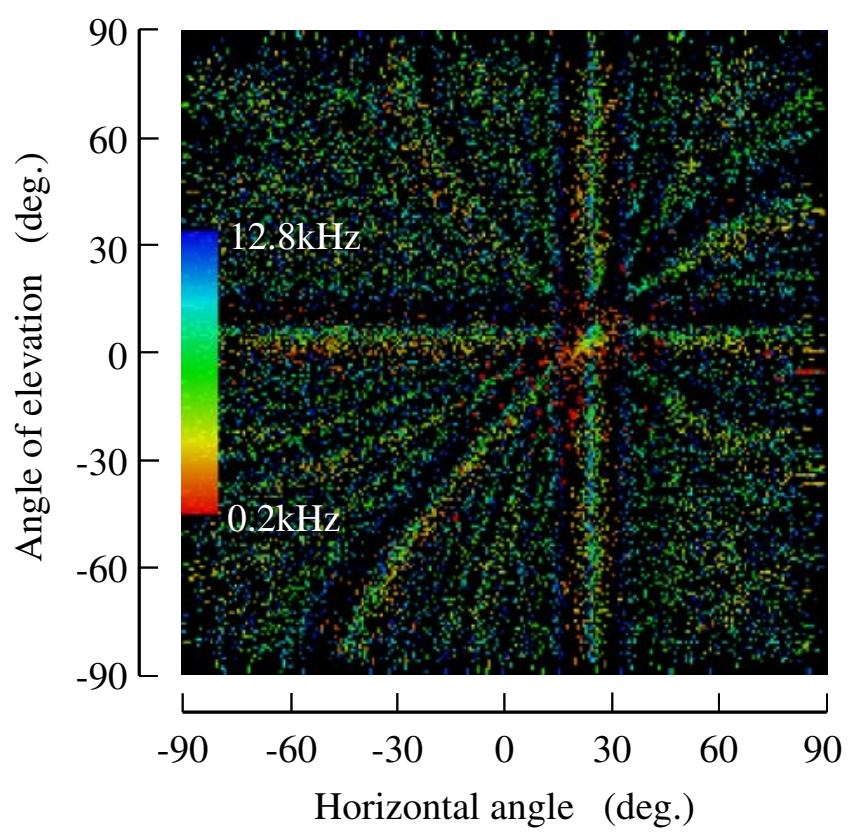

Fig. 9 2D image of colorful point sources equivalent to a single two-way loudspeaker for horizontal angle +27 degrees and angle of elevation +6 degrees $(D=1 \mathrm{~m}$, $S=0.20 \mathrm{~m})$.

\subsection{D Source Images for Loudspeaker at Various Positions}

In this section, 2D colorful source images were identified for a single two-way loudspeaker positioned off-center both horizontally and vertically. To clarify whether the broadband sound source can be visualized from all directions spherically, we consider two cases of positioning the loudspeaker off-center.

First, a single two-way loudspeaker was placed at a horizontal angle of +27 degrees and an angle of elevation of +6 degrees at a distance of $1 \mathrm{~m}$. Figure 9 shows the 2D colorful source image for the loudspeaker. A point source convergence was observed at approximately the true loudspeaker position. The false source distributions were not symmetrical to the origin, unlike those of the centerpositioned loudspeaker shown in Fig. 7.

Second, the 2D colorful source images for a fixed loudspeaker were identified for the cases where the microphones were horizontally rotated step by step around their position, as shown in Fig. 10. Since the coordinate axis had fixed microphones, the fixed loudspeaker appeared to rotate inversely to the source image. The distance between the loudspeaker and the microphones changed from $1 \mathrm{~m}$ to approximately $1.76 \mathrm{~m}$ during rotation. Figure 11 shows several 2D source images observed while the microphones were rotated horizontally around their position. The rotation angles are based at the rotated coordinate axis. Numerous point sources converged approximately at the loudspeaker position, except at the 


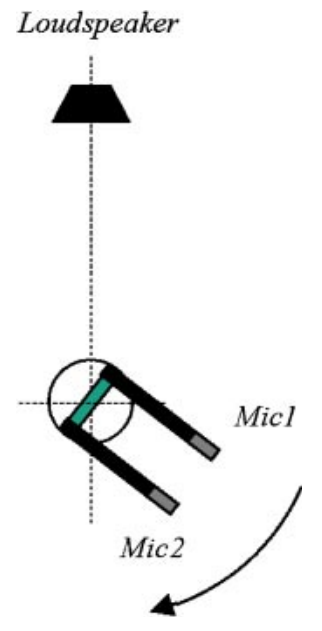

Fig. 10 Rotation of microphones horizontally around their position.

rotated angle of +96 degrees, shown in Fig. 11(b). The amplitude of the identified angle decreased and showed a deviation of approximately 180 degrees from true angle for angles greater than 90 degrees. All of the false source distributions showed geometrical patterns that were approximately symmetrical to the $x$-axis, as shown in Fig. 1. The 2D source image directly behind the microphones, i.e., a rotated angle of 180 degrees, is shown in Fig. 11(c). The microphone stems and their supports appeared to block direct sound propagation when the speaker was positioned further back. This condition is outside of the hypothesis in our model that sound propagates straight without diffraction. The source image was more obscure and pale due to scattered high-frequency tones, compared with that of the front loudspeaker in Fig. 7. Even for the case in which the loudspeaker was located directly behind the microphones, the true position of the loudspeaker could be determined using the cues of the colorful point source convergence. These results indicate that by using CAVSPAC, the 2D image can be identified for the broadband sound source from all directions spherically, except in the area just beside, above and under the microphones.

\subsection{True Source Image}

The false sources are eliminated by extracting one point source from the many identified at each frequency. We reconsider the 2D source image for the front loudspeaker at a distance of $3 \mathrm{~m}$, shown in Fig. 8. This image includes not only true point sources, but also the false point sources that are generated owing to "phase ambiguity." In this case, the true point source location was estimated by determining both the horizontal angle and the angle of elevation nearest to zero degree at each frequency, since the loudspeaker was set approximately on the $y$-axis, as shown in Fig. 1 . Figure 12 shows the 2D colorful image of true point sources for the front loudspeaker after eliminating the false

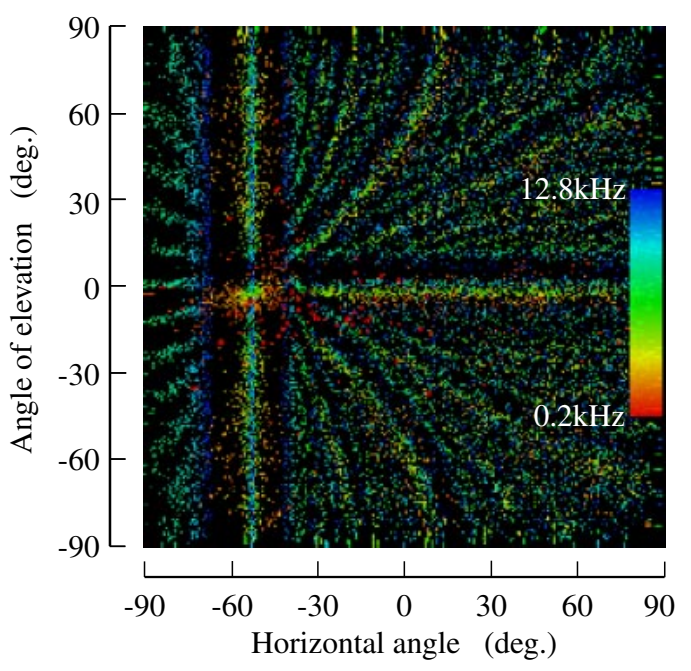

(a) +53 deg., $\mathrm{D}=1.09 \mathrm{~m}$

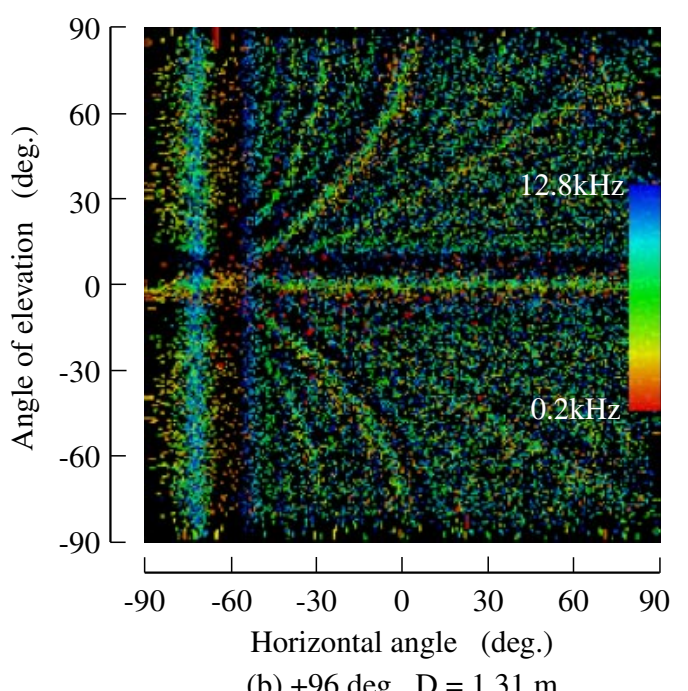

(b) +96 deg., $\mathrm{D}=1.31 \mathrm{~m}$

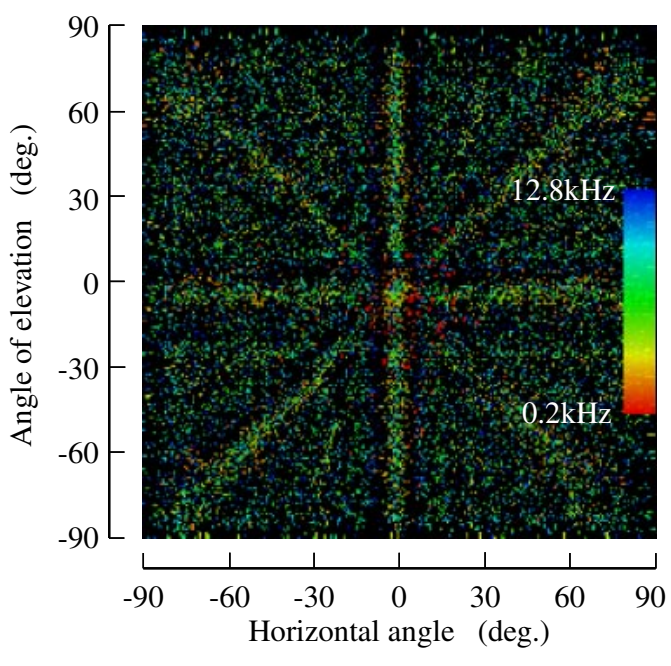

(c) +180 deg., $\mathrm{D}=1.78 \mathrm{~m}$

Fig. 11 2D images of colorful point sources for a single two-way loudspeaker when the microphones are horizontally rotated step by step around their position. Rotated angles are (a) +53 degrees, $D=1.09 \mathrm{~m}$, (b) +96 degrees, $D=1.31 \mathrm{~m}$ and (c) +180 degrees, $D=$ $1.78 \mathrm{~m}$ on the rotated coordinate axis $(S=0.20 \mathrm{~m})$. 


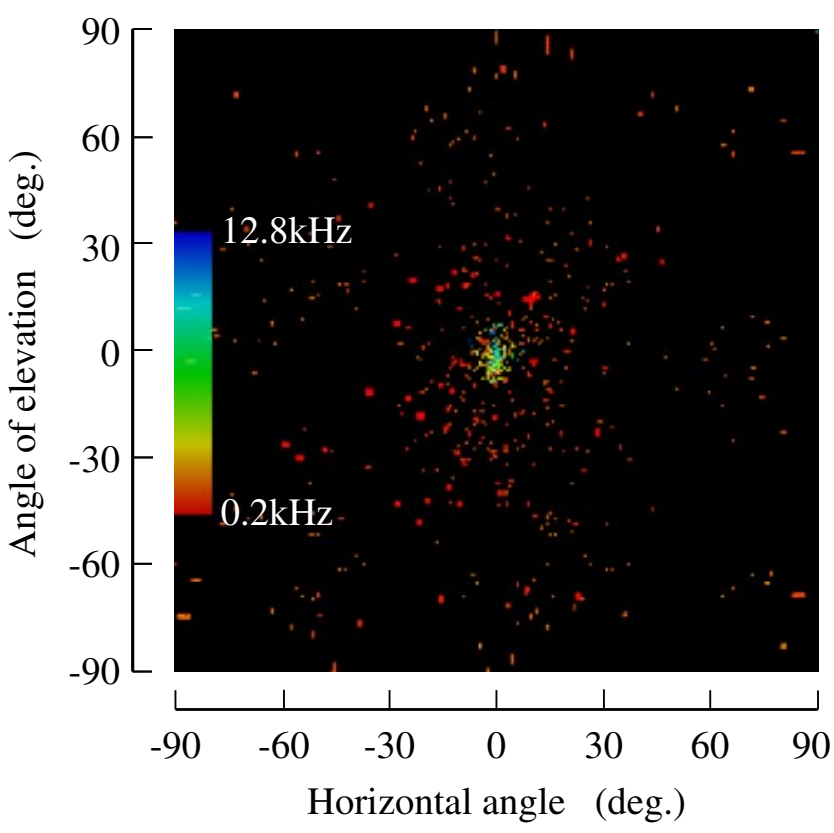

Fig. 12 2D colorful image of true point sources equivalent to a single two-way loudspeaker corresponding to Fig. $8(D=3 \mathrm{~m}, S=0.20 \mathrm{~m})$.

point sources. In particular, several point sources that ideally radiated low-frequency tones were distributed over a relatively wide range on all sides of the loudspeaker position. This corresponds to the random distribution of the source angle at frequencies lower than approximately $2 \mathrm{kHz}$, as shown in Fig. 6. Most true point sources that ideally radiated higher frequency tones converged close to the loudspeaker position. This image does not depend on the sound pressure level, since phase does not depend on the sound pressure level with regard to being precisely detectable.

\subsection{Comparison of Colorful Source Images Obtained under Different Microphone Intervals}

In this section, we present the benefit of CAVSPAC by comparing the colorful source images for the same loudspeaker for two different microphone intervals. The loudspeaker was set at a distance of $3 \mathrm{~m}$. The sampling rate was $16.4 \mathrm{kHz}$. The frame period was set to $500 \mathrm{~ms}$. Ten measurements were averaged. The colors were assigned to the frequency tones over the frequency range from $0.2 \mathrm{kHz}$ (red) to $6.4 \mathrm{kHz}$ (blue). Figures 13(a) and 13(b) show the 2D source images obtained with the two different microphone intervals of (a) $0.04 \mathrm{~m}$ and (b) $0.20 \mathrm{~m}$. The interval $0.04 \mathrm{~m}$ is shorter than the sound wavelength over the full measuring frequency range, so that the source position can be unambiguously identified at each frequency. The point sources were scattered widely around the loudspeaker position in Fig. 13(a). In contrast, phase ambiguity confusion occurs at frequencies from $1.7 \mathrm{kHz}$ to $6.4 \mathrm{kHz}$ in

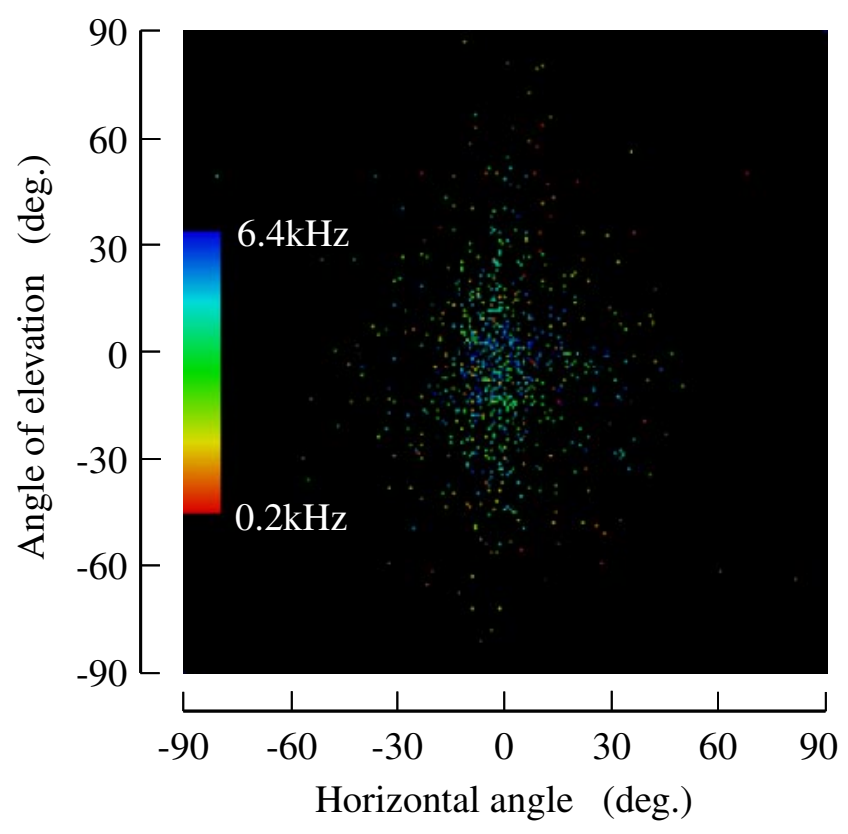

(a) $\mathrm{S}=0.04 \mathrm{~m}$

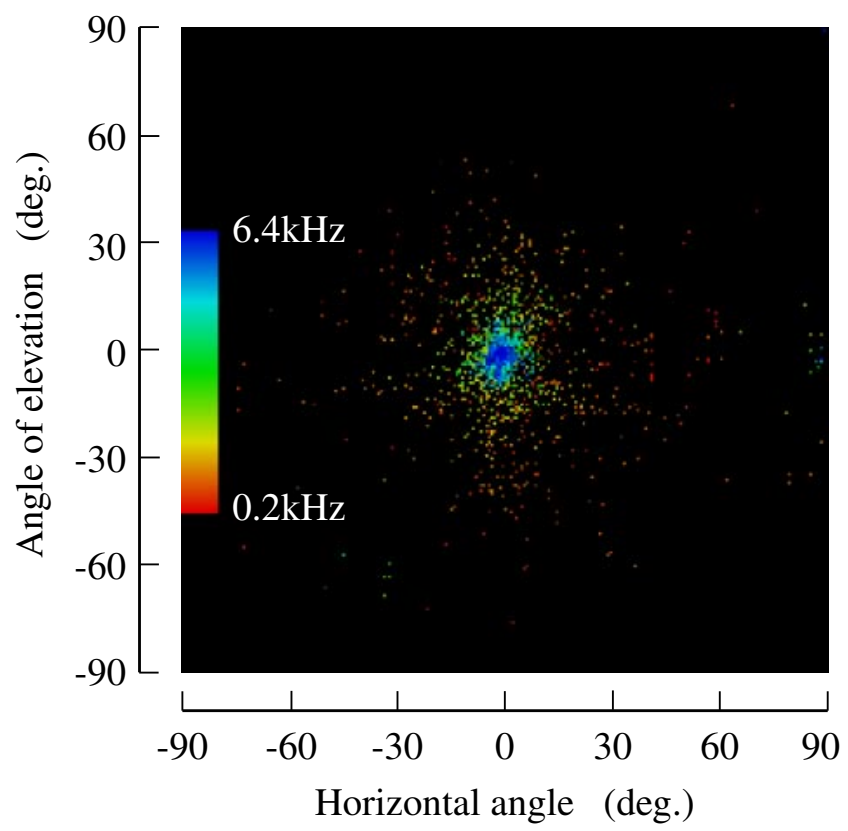

(b) $\mathrm{S}=0.20 \mathrm{~m}$

Fig. 13 2D colorful images of true point sources equivalent to the same two-way loudspeaker under different microphone intervals: (a) $S=0.04 \mathrm{~m}$ and (b) $S=0.20 \mathrm{~m}$. Colors are assigned to the frequency tones from $0.2 \mathrm{kHz}$ to $6.4 \mathrm{kHz}(D=3 \mathrm{~m})$.

Fig. 13(b). The 2D colorful source image represents the loudspeaker position more clearly at higher frequencies. Figures 14(a) and 14(b) show the distribution of the normalized number of times on the horizontal source angle, counting the number of data at each source angle over the full frequency range in Figs. 13(a) and 13(b), respectively. The main peaks appeared correspond to the loudspeaker position in both figures. Numerous point 


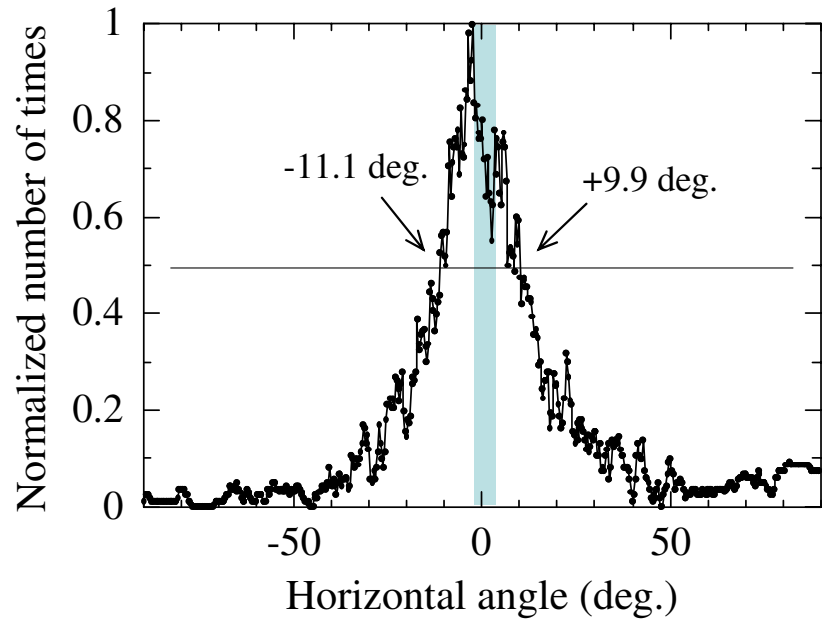

(a) $\mathrm{S}=0.04 \mathrm{~m}$

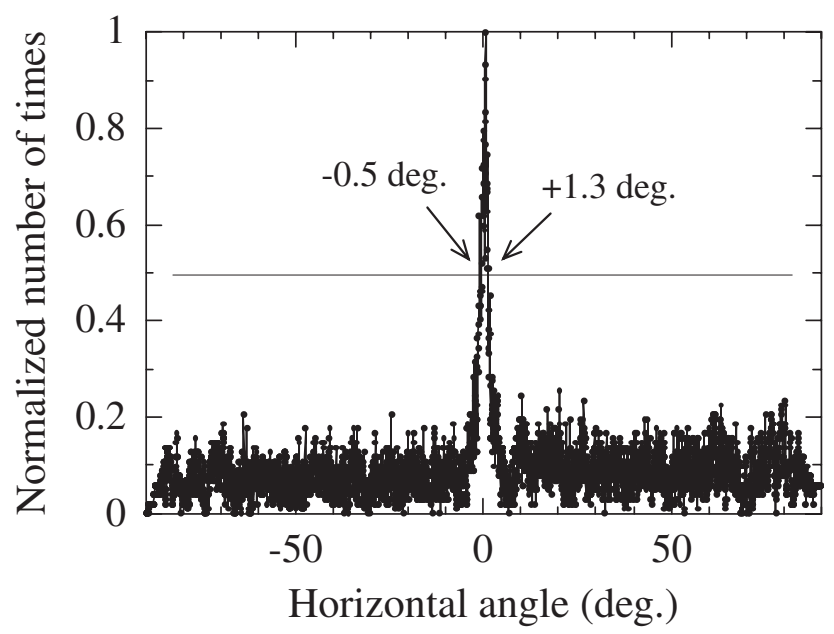

(b) $\mathrm{S}=0.20 \mathrm{~m}$

Fig. 14 Number of times of distributions on horizontal angle of 2D images under two different microphone intervals, (a) $S=0.04 \mathrm{~m}$ and (b) $S=0.20 \mathrm{~m}$, corresponding to Figs. 13(a) and 13(b) $(D=1 \mathrm{~m})$.

sources were distributed in the angle ranges from -11.1 degrees to +9.9 degrees in Fig. 14(a) and from -0.5 degrees to +1.3 degrees in Fig. 14(b), when the number of times of the horizontal source angle exceeded the threshold value of 0.5 . The angle for the woofer is estimated to be 1.7 degrees by geometry. A phase difference larger than 360 degrees can be utilized in obtaining the image by solving the "phase ambiguity" confusion at high frequencies. It should be emphasized that the moderate wider microphone interval than the wavelength leads to a better resolution of the source image.

\section{CONCLUSION}

Computational acoustic vision by solving phase ambiguity confusion (CAVSPAC) was proposed for 2D colorful imaging on the broadband sound environment. The 2D distributions of equivalent point sources were identified as images from the ambiguous cross-power spectral phase using two pairs of microphones. Each point source was assigned a color corresponding to the frequency. The 2D location of the true point source was identified as the combination of two angles nearest to the frequencyindependent angles.

The results can be summarized as follows. First, the source images representing clustered point sources exhibited several patterns: numerous colorful point sources converged near the true loudspeaker position and several thick and thin lines corresponding to false point sources were radially distributed from the loudspeaker position. Second, using CAVSPAC, the 2D image could be identified for the broadband sound source from all directions spherically, except in the area just beside, above and under the microphones. Finally, the moderate wider microphone interval than the sound wavelength led to a better resolution of the source image.

It may be possible to adapt CAVSPAC to higher frequencies up to the $\mathrm{GHz}$ band of sonic and electromagnetic waves owing to the nature of these waves in order to visualize several wave fields. One of next targets will be real-time $2 \mathrm{D}$ imaging as pointillisme on various wave fields, similarly to on an acoustic field.

\section{ACKNOWLEDGEMENTS}

R. Shimoyama would like to express his sincere gratitude to Professor Hideo Suzuki of Chiba Institute of Technology and Professor Hideo Shibayama of Shibaura Institute of Technology for fruitful discussions in 2001. This research was supported by Matching fund subsidy from the Ministry of Education, Culture, Sports, Science and Technology (MEXT), 2000-2005.

\section{REFERENCES}

[1] A. S. Bregman, "Auditory scene analysis: Hearing in complex environment," in Thinking in Sound, S. McAdams, E. Bigand, Eds. (Oxford University Press, Oxford, 1993), Chap. 2, pp. 1036.

[2] F. L. Wightman and D. J. Kistler, "Sound localization," in Human Psychophysics, W. A. Yost, A. N. Popper, R. R. Fay, Eds. (Springer-Verlag, NewYork, 1993), pp. 155-193.

[3] W. Lindemann, "Extension of a binaural cross-correlation model by contralateral inhibition. I. Simulation of lateralization for stationary signals," J. Acoust. Soc. Am., 80, 1608-1621 (1986).

[4] M. Rucci and J. Wray, "Binaural cross-correlation and auditory localization in the barn owl: A theoretical study," Neural Net., 12, 31-42 (1999).

[5] M. Rucci, J. Wray and G. M. Edelman, "Robust localization of auditory and visual targets in a robotic barn owl," Robot. Auton. Syst., 30, 181-193 (2000).

[6] C. Liu, B. C. Wheeler, W. D. O’Brien, Jr., R. C. Bilger, C. R. Lansing and A. S. Feng, "Localization of multiple sound sources with two microphones," J. Acoust. Soc. Am., 108, 1888-1905 (2000).

[7] R. Shimoyama and K. Yamazaki, "Acoustic source local- 
ization using phase difference spectrum images," Acoust. Sci. \& Tech., 24, 161-171 (2003).

[8] J. L. Flanagan, D. A. Berkley, G. W. Elko, J. E. West and M. M. Sondhi, "Autodirective microphone systems," Acustica, 73, 58-71 (1991).

[9] E. O. Belcher, W. L. J. Fox and W. H. Hanot, "Dual-frequency acoustic camera: A candidate for an obstacle avoidance, gapfiller, and identification sensor for untethered underwater vehicles," Ocean'02, pp. 2124-2128 (2002).

[10] Y. Wang, J. Li, P. Stoica, M. Sheplak and T. Nishida, "Wideband RELAX and wideband CLEAN for aeroacoustic imaging," J. Acoust. Soc. Am., 115, 757-767 (2004).

[11] N. Kamiakito, H. Nogami, D. Tominaga, Y. Yamashita, T. Zaiman, M. Owaki, T. Sugiyama and H. Wada, "Development of noise source identification system with multi-microphone sound signal process enhanced by visual image process - A study on decrease of reflection influence," J. Archit. Plann. Environ. Eng., 564, 1-7 (2003).

[12] M. Konishi, "Study of sound localization by owls and its relevance to humans," Comp. Biochem. Physiol. A, 126, 459469 (2000).

[13] R. Shimoyama and K. Yamazaki, "Multiple acoustic source localization using ambiguous phase differences under rever- berative conditions," Acoust. Sci. \& Tech., 25, 446-456 (2004).

[14] P. V. C. Hough, "Method and means for recognizing complex patterns," U.S. Patent 306965 (1962).

[15] R. E. Duda and P. E. Hart, Pattern Classification and Scene Analysis (Wiley, NewYork, 1973).

Ryuichi Shimoyama received B.E. and M.E. degrees in Electrical Engineering from Toyama University and Ph.D degree in System Science from Okayama University, in 1979, 1981, 1994, respectively. He was an invited researcher at Center for Intelligence System of Vanderbilt University, USA and Polo Sant'Anna Valdera of Pisa University, Italy, from 2005 to 2006 . He is a professor of Nihon University in Japan. His current research interest includes artificial intelligence on auditory system and robotics. He is a member of IEICE, IPSJ, ASJ and JSST.

Ken Yamazaki received Dr.Sci. degree from Okayama University, Japan, in 1993. He is currently a Professor at Nihon University in Japan. He investigated in Sothampton University UK as a guest researcher by 1992 since 1989. His research interests are the visualization and numerical simulation of the sound field, the creation of the comfortable sound space et al. He is a member of ASJ, JSST, IEICE, AIJ in Japan and ASA in USA. 